\title{
Experimental and Numerical Study of Sewing Seams of Automobile Seat Covers Under Unidirectional and Multiaxial Loading
}

\author{
Natalia Kovalova ${ }^{1}$, Petr Kulhavý ${ }^{2}$, Josef Vosáhlo ${ }^{2}$, Antonin Havelka ${ }^{1}$ \\ ${ }^{1}$ Technical University of Liberec, Faculty of Textile Engineering, Studentska 2, 46117 Liberec 1, Czech Republic \\ ${ }^{2}$ Technical University of Liberec, Institute for Nanomaterials, Advanced Technologies and Innovation, Studentská 2, 46117 Liberec 1, \\ Czech Republic
}

Corresponding Author: Natalia Kovalova, natalia.kovalova@tul.cz

\begin{abstract}
In industrial textiles, knowing the exact characteristics and behaviour of materials is important. For car seats, industrial textiles not just cover the underlying foam but also increase rigidity of the seat cushion and influence viscoelastic behaviours of foams. Moreover, strength of sewn seams is one of the main quality parameters. Herein, four polyester and polyamide threads were sewn on a material used for car seat covers through lockstitch sewing. Combinations of these materials were studied using static tests in the unidirectional and multiaxial variants. The experimental measurements recorded using a high-speed camera and computer tomography were used to create CAD models. Numerical simulations were conducted using these models and the obtained material models. These model studies help predict and describe the stresses emerging within various types of textile and the threads in their connections. The simulation results agree well with the experimental results.
\end{abstract}

\author{
ARTICLE HISTORY \\ Received: 11.12.2018 \\ Accepted: 01.10.2019

\section{KEYWORDS} \\ sewing seam; strength, 3D \\ curves, parts contact, \\ numerical model
}

\section{INTRODUCTION}

Automotive textiles represent the most valuable market worldwide for technical textiles. Within this segment, a broad spectrum of products having novel textile structures with performance properties and attractive design is available [1]. Car seat covers are perhaps the most familiar automotive textiles. Considerable technical input is necessary to develop materials that must withstand rigorous use (and abuse) and yet last for the lifetime of the car seat [2].

The exact knowledge about mechanical properties of the used materials is the basic prerequisite for successful product construction. The mechanical properties of materials play a crucial role in the control of production technology, quality control and development of new materials. In addition, in a few cases, human life may be placed in danger owing due to a malfunction of these products (such as parachutes, airbags, and safety belts). Therefore, it is necessary to understand not only the strength characteristics of textile materials and threads but also the performance of seams.

The seam characteristics include strength, elasticity, durability, safety, and appearance. Inconsistencies in these characteristics can lead to significant differences in seam behavior and can affect seam deformation characteristics. These characteristics originate from four principal sources: speed, needle, thread, and fabric. It has been found that fundamental interactions among these factors exist [3].

Mazari et. al. [4] confirmed that the friction between the needle and sewing thread is one of the major sources of

To cite this article: Kovalova N, Kulhyvy P, Vosahlo J, Havelka A. 2019. Experimental and numerical study of sewing seams of automobile seat covers under unidirectional and multiaxial loading Tekstil ve Konfeksiyon 29 (4),322-335. 
needle heating. High needle temperature during sewing weakens the thread because thread tensile strength is a function of temperature as well, and this leads to decreased production. In addition, the strength of the final stitched thread is $30-40 \%$ lower than that of the parent threads. A very high needle temperature can damage the materials as well, for example, a few synthetic fabrics or plastics that come into direct contact with the needle during sewing. Zak [5] described the principles of mutual fabric interaction by using Pierce's model and numerically by using the finite element method (FEM).

According to Midha [6], during high-speed sewing, the strength of sewing threads decreases substantially. Any change in thread strength is closely connected to the thread's passage over the guiding elements of the sewing machine and, consequently, the friction and bending originating between the needle's thread and the touching areas, as well as friction with the bobbin thread [7]. These pressure and friction forces result from internal tension.

One of the major quality factor associated with seams is fabric type. At present, different types of materials are used for upholstering car seats. These include technical textiles for car seat covers, such as woven, knitted, and nonwoven fabrics; natural and artificial leather from synthetic polymers such as polyester, polyamide, and viscose; and blends of wool and cotton with synthetic fibres. Woven and knitted structures are two main fabric forming methods used for manufacturing car seat upholstery manufacturer [8]. Ujevic et al. [9] studied the influence of fabric type on the mechanical properties of the seam, and they found that technical woven fabrics have higher breaking force and penetration resistance than knitted fabric, but knitted fabric is softer and more elastic. Sewing thread accounts for a small percentage of the final product cost but accounts for $50 \%$ of the seam strength. Hence, thread plays an important role in seam quality, especially in car seat covers, where it is necessary to consider the following factors: thread fiber type, thread construction, thread finish, and thread size.

For creating a high-quality seam it is necessary to consider all abovementioned parameters. This is a fairly timeconsuming process because of the wide variety of existing materials. Therefore, the aim of this study is to develop a numerical model of sewing seams considering all necessary parameters. When studying literature and the latest world research, it is possible to find some numerical models that relate to the textiles connections, however they are simplified by local homogenization of the entire seam as one local area with usually orthotropic properties or some studies of just small segment of the yarn. No model that interprets the thread in its entirety, including individual yarn twists and holes in the fabric has been found.

There are many types of fabric constructions used in automobiles as seat cover fabrics. One type of twill fabric and four types of sewing threads were used for determination of the mechanical properties with using different measuring methods. Together with that, twill fabric type and one type of polyamide sewing thread were used for creating numerical model of sewing seam. The twill fabric used is the most common fabric used by researchers and its possible to compare with the findings of the previous researchers. In future the numerical model can be tested for multiple textile fabrics.

The required material properties have been measured using a designed multiaxial apparatus. Seam strength was determined by conducting the standard uniaxial static experiments. However, because automobile seat covers are subjected to various loadings, especially, multi-axis load, a special testing device was designed and constructed $[3,10]$. This device operates on the principle of extrusion of a spherical cap into a cylindrically clamped sample. Each type of test was recorded on a high-speed camera and the samples before and after testing were screened using computer tomography to obtain precise geometric parameters of the created CAD model. Based on this CAD geometry and by using the commercial software ANSYS, several nonlinear contact numerical simulations were conducted to predict the behaviour of the stitched seams instead of the classical experiments [11]. Therefore, the main aim of this article is to thoroughly describe the behaviour of stitched seams for various textile materials and various loading conditions, as well as to develop the corresponding simulation model that can predict the real behaviour of a selected combination of threads and fibres instead of having to conduct expensive experiments.

\section{MATERIAL AND METHOD}

\subsection{Material}

During the process of product usage, sewn seams and materials are subjected to variable loads, leading to various deformations. For textile materials, which show considerable anisotropy or orthotropy, testing along one axis is generally insufficient, and it is necessary to load two axes simultaneously. According to Quaglini [12], because of the small thickness of fabric, it is usually a neglected component of strain along the thickness direction of the material, and technical textiles are mainly thought to be affected by plane stress $[13,14]$.

\subsubsection{Threads}

To investigate the effect of different sewing threads on seam strength, four different threads were used for specimen preparation (Table 1). Threads of Type B, Type $\mathrm{C}$, Type $\mathrm{H}$, and Type M1 were used to investigate seam strength under multiaxial loading. Other types of threads were used for static measurements. These threads are commonly used in the industry to sew car seat covers. According to ISO 2060 [15] and ISO 2061 [16], the values of fineness and twist of the sewing threads were investigated experimentally. 
For preparing all samples tested in this study, seam parameters were selected precisely, and the sewing machine was set up accordingly. A Dürkop Adler sewing machine with an upper thread tension of $4 \mathrm{~N}$ and lower thread tension of $1 \mathrm{~N}$ was used. Classes of seams and stitching are defined in the international standards ISO 4916 and ISO 4915 respectively. The parameters of the sewing process were as follows: superimposed seam with width of $10 \mathrm{~mm}$ and 301 Lockstitch with a density of $3.3 \mathrm{~mm}$. These parameters of stitches and seams are most commonly used for sewing car seat covers.

\subsubsection{Fabric}

Sandwich-type fabrics are used for preparing samples to test the mechanical properties of sewing seams under different type of loading. The material consists of the PES face fabric, foam core and the weft knit fabric as the back. Material thickness was defined using the method given in the standard ISO 5084 [17]. The exact parameters of the fabric are listed in Table 2.

\subsubsection{Experimental samples for unidirectional and multiaxial loading}

The samples for uniaxial loading were prepared according to the norm CSN EN ISO 13935-1 [18]. Laboratory samples of size $700 \mathrm{~mm} \times 350 \mathrm{~mm}$ were prepared of the material used to industrial manufacturing of car seat covers (Figure 1a). The samples were folded, such that the edge of the fold was parallel to the shorter side of the sample. The samples were prepared in the warp and the weft directions. Finally, the real width of the sample was $50 \mathrm{~mm}$, and the length of the sample used for investigation was $330 \mathrm{~mm}$ (Figure 1b).

The production of experimental samples for measuring seam strength under multiaxial stress begins in the same way as that for uniaxial stress. Parts of the fabric are sewn together, and the remaining parts are prepared for each set of experiment on the test samples by cutting out the desired shapes (Figure 1b). The shaded area in Figure 1b shows the portion of the test sample on which the spherical cup is pushed [19].

b)
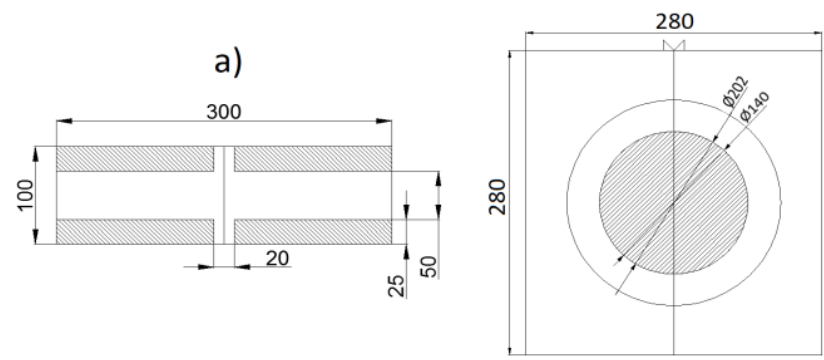

Figure 1. Laboratory specimens: a) The UD sample and b) experimental sample

\subsection{Experimental Devices and Methods}

As Reinhardt pointed out [20], in terms of textile testing, the materials are almost always loaded and should be tested biaxially. According to Novak and Hanus [21], anisotropic materials such as the tested coating materials show noticeable hysteresis and different deformations according along different loading directions owing to specific mechanical properties. In tensile tests of textiles along one axis in the longitudinal direction, the main stress concentrators are formed, and the fabric subsequently ruptures near the clamping jaws. By contrast, the tension along the transverse direction is more uniformly concentrated at the sample centre.

According to Escárpita [22], testing of multiaxial loaded samples is necessary to ensure that the test device meets the basic requirements. There must be strictly tension or compression, and spurious shear or bending loads must be avoided. Generally, the use of a hydraulic or mechanical system comprising linear motors, cable system, pulleys, and bearings is recommended.

Table 1. Properties of threads used in this study

\begin{tabular}{lllll}
\hline Property & Type B & Type C & Type H & Type M1 \\
\hline Tread content & PA6.6 & PA6.6 & PES & PES \\
Actual thread density T [Tex] & 107.8 & 79.0 & 94.8 & 78.7 \\
Twist [t/m] & 347 & 409 & 375 & 416 \\
\hline
\end{tabular}

Table 2. The fabric specifications

\begin{tabular}{lll}
\hline \multicolumn{1}{c}{ Parameter } & \multicolumn{1}{c}{ Value } \\
\hline Width $[\mathrm{mm}]$ & $1800 \pm 20$ & Weave type \\
\hline Weight $[\mathrm{g} / \mathrm{m} 2]$ & $490 \pm 50$ & \\
\hline Thickness $[\mathrm{mm}]$ & $4.5 \pm 0.5$ & \\
\hline Face $[\mathrm{g} / \mathrm{m} 2]$ & $330 \pm 30(100 \%$ PES $)$ & \\
\hline Lamination & Polyester foam & \\
\hline Backing $[\mathrm{g} / \mathrm{m} 2]$ & $38(100 \%$ PES $)$ & Twill $(2 / 1)$ weave \\
\hline
\end{tabular}


In the tests, a sample of certain dimensions with the seam in the middle is stretched in a direction perpendicular to the seam at a constant rate until seam breakage.

\subsubsection{Unidirectional tests}

The most common tests applied to automotive seat upholstery were specified by the Society of Automotive Engineers (SAE) and the American Society for Testing Material (ASTM) [23]. For determining the mechanical properties associated with seam sewing, the Strip tests [18] are used. For these experiments, it was necessary to select and condition the samples according to the standard ISO 139 [24].

During the static tests, the maximum force for seam rupture is determined using the strip method when the applied force is perpendicular to the seam. In the tensile test that is a part of this method, the entire width of the test specimen is gripped in the jaws of the testing machine (Figure 2). The feed speed of the jaws was set to $100 \mathrm{~mm} / \mathrm{min}$ [18].

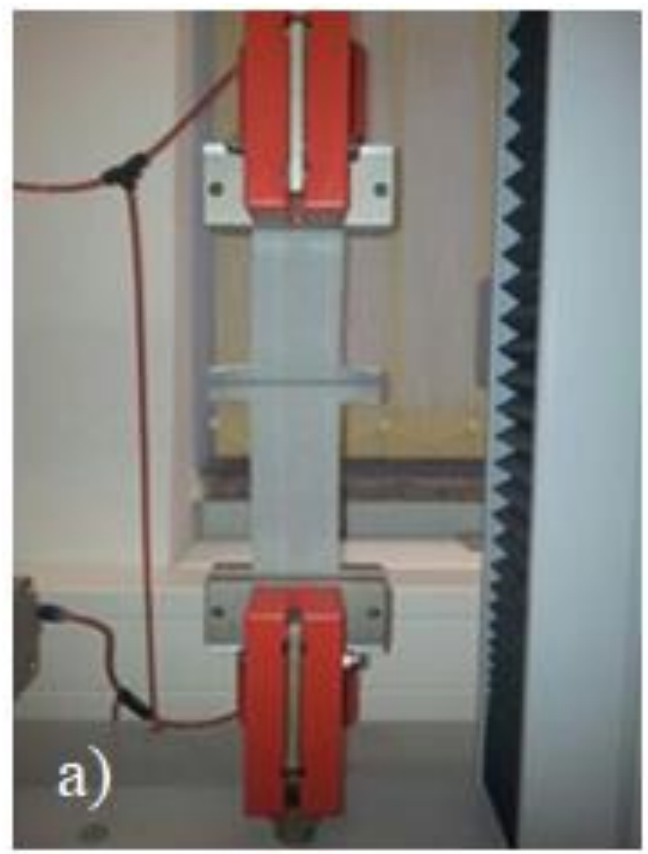

Figure 2. Experimental device for unidirectional texting

\subsubsection{Multiaxial testing}

A device that can be used to measure sewing seam strength under multiaxial stress has been developed (Figure 3a, 3b). The device consists of a spherical cup (1), an upper jaw (2), a lower jaw (3), screws (4), a hook clamp (5), light source (6), and cylindrical frame (7). In this case, the essential requirement is applied pressure at seam sample, and therefore, it is necessary to choose appropriate settings of the tensile machine. A laboratory-made seam sample was clamped between the two circular jaws and fixed using hook clamps. Then, during the tests, the indenter was moved vertically downward toward the seam connection at a constant velocity of $100 \mathrm{~mm} / \mathrm{min}$.
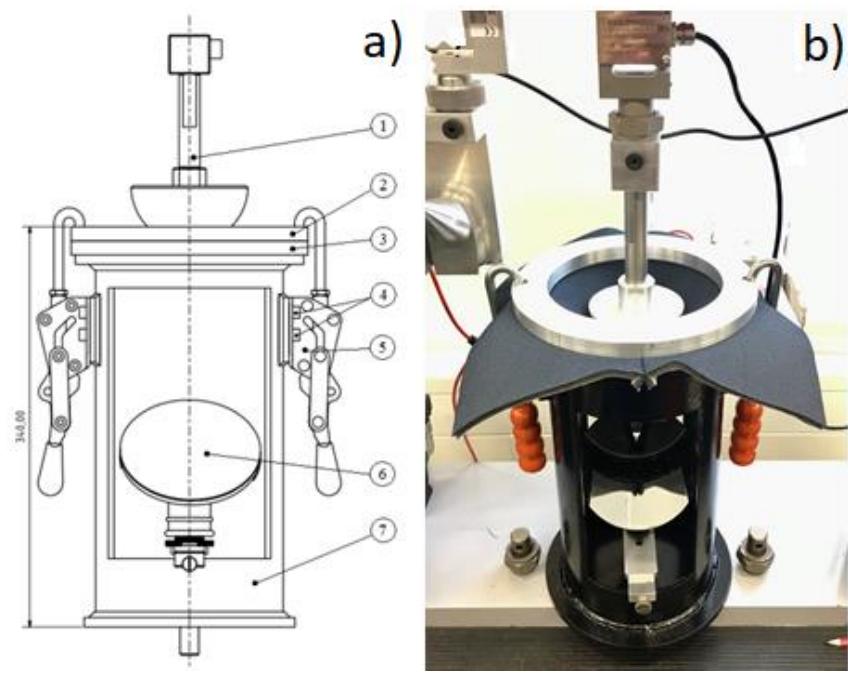

Figure 3. Laboratory instrument for measuring seam strength under multiaxial stress: a) Schematic view of the created device and b) photograph of constructed device

\subsection{Model Creation}

Given the fact that using conventional imaging devices, it is not possible to simply show the mutual thread connections, the computer tomography (Figure 4a, Figure 4b) was used. However, it is not possible to simply convert the obtained graphical data into a CAD-compatible format. Therefore, the images were used mainly as shape templates. Simultaneously pictures obtained using a macro camera was used to measure the fundamental dimensions of the seam.

\subsubsection{CAD model}

For creating the models, the Creo software application was used. Creo is the one of the only few CAD modellers that allows supports direct manual and parametric input for creating 3D curves, including their transition parameters and enhanced control of their base polygons [25].

For creating so complex CAD models, it is important to find the most appropriate forming and carrier curves that can respond to sudden changes in the direction vectors and radius of curvature. Generally, it is possible to express a curve in space by using the explicit expression $\mathrm{z}=\mathrm{f}(\mathrm{x}, \mathrm{y})$ or implicitly by using $\mathrm{F}(\mathrm{x}, \mathrm{y}, \mathrm{z})=0$. In computer graphics, the parametric curves are expressed by using the point equation $P(t)=[x(t) ; y(t) ; z(t)]$ or with the steering vectors $\vec{p}(t)=[x(t) ; y(t) ; z(t)][26]$.

The design concept was compiled using B-splines and the Bezier curve (Figure 5). A B-Spline is a generally controlled modification of piecewise polynomial functions and their approximations. It is in fact a curve that passes 
through the specified points, while seeking to achieve the lowest values of the radii of curvature in each part. By contrast, the Bezier curve is firmly defined by the end and start points, which create the so-called control polygon and other (internal) points that indicate and allow for direct control of the internal curvature. Currently, the Bezier plate is one of the most popular curves used, for example, in various font styles [26, 27]. By changing the magnitudes of the Bezier curve parameters, it is possible to obtain and check, for example, the main characteristics of lines, ellipses, parabolas, and hyperbolas. The common feature of the two curves is that by changing any one point, the entire curve can be varied. a)

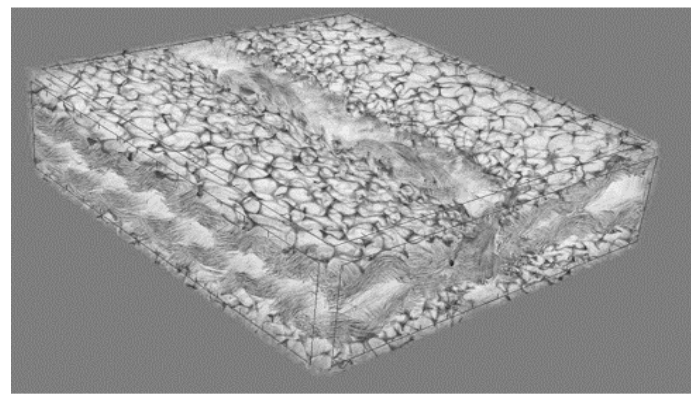

b)

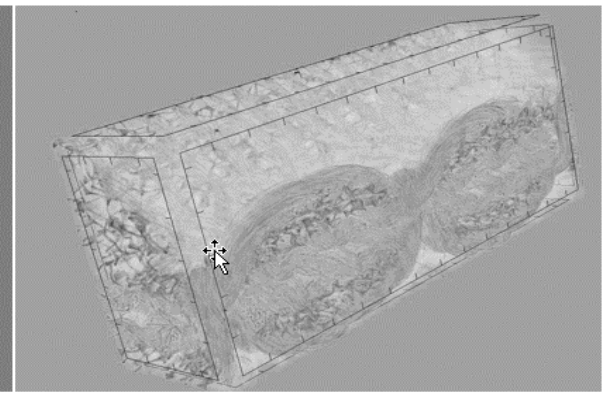

Figure 4. Example of CT scan of seam: a) Internal and b) external view of seam
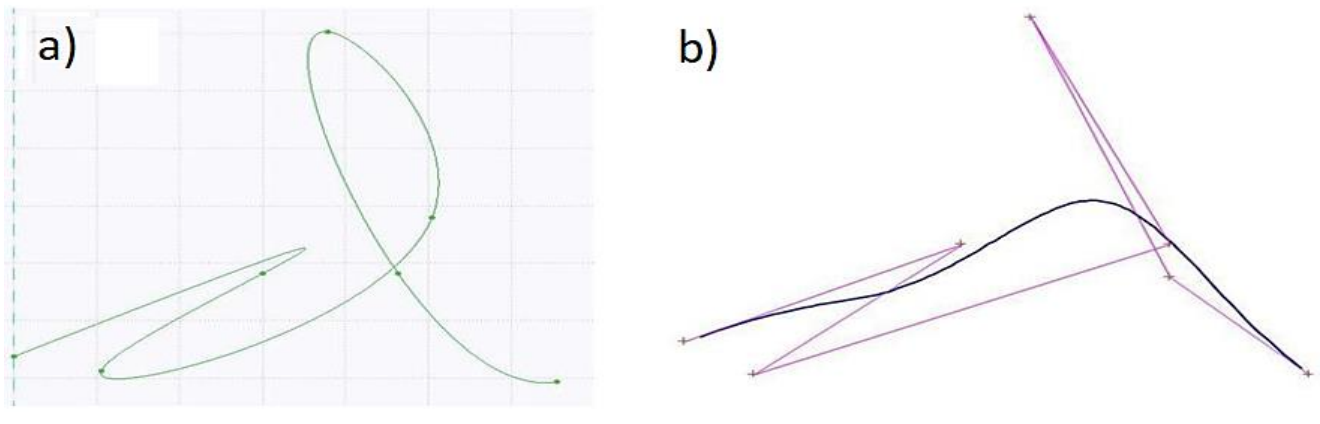

Figure 5. Same points interlayered using a) a spline and b) the Bezier curve

The spline curve has been found to be unsuitable for the thread model, Possible because during model creation, the individual parts are connected together, and the curve is sensitive to any local change. This could cause unwanted changes to the entire geometry, even when only a tiny local variation occurs. This leads to interference among the assembly parts and possibly to errors during FEM computation. The Bezier curve was found to be suitable because of firmly specified start/finish points and because the curvature is independent of the adjacent connected segments. For a required connection, it is possible to simply use the adjustable parameters of normality, tangentiality, or parametric settings of the transitions. A very important parameter of the model is the setting of the parametric classes of the continuity transitions between individual segments that define the continuous derivatives in the three classes. Connection $\mathrm{C} 0$ refers to a common point with two arbitrarily passing vectors and two curves of different radii of curvature. Continuity $\mathrm{C} 1$ is characterized by one common tangential vector $\vec{t}_{1}$ at the transition point, but two curves having different radii of curvature. Completely analogously, class $\mathrm{C} 2$ is characterized by a common tangent vector and constant value of the curvature at the transition point.

At first, the yarns (Figure 6a) were interconnected. For connection of the yarns, the merged surfaces were used, and the whole geometry was formed using the assembly cut-off with the offset surfaces (Figure 6b). The CAD model of the entire assembly was verified by tracking global interferences of all parts, where any intersections could not occur. Checking the geometry of the entire assembly this way was necessary, especially for solving the future numerical contact model. The assembly of mutual thread connections and implementation on fabric are shown in Figure 7c. 
a)

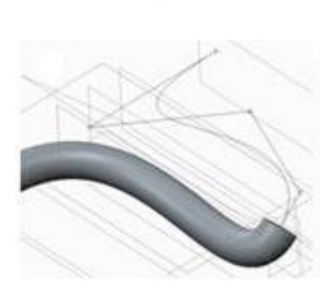

b)

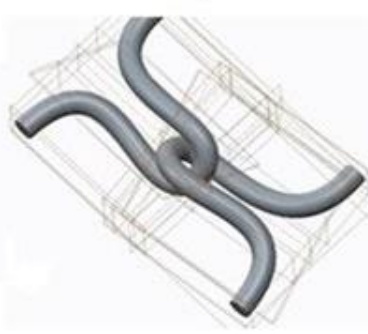

c)

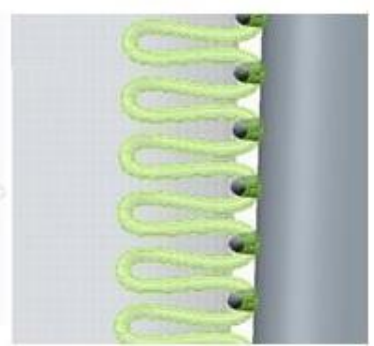

Figure 6. Model of a) thread and b) binding point of upper and lower thread c) assembly of mutual threads connection and implementation on fabric

\subsection{Numerical Model Theory}

An appropriate choice of a material model is very important. For the materials used in this study, it was necessary to determine the elastic and shear moduli [GPa], tensile strength [GPa], and set the Poisson ratio [-] by conducting various experiments. For creating the numerical model of the fabrics, the spatial ten square node tetrahedron SOLID 187, which is suited for modelling irregular structures (created, e.g., in CAD systems), was used. It is defined by eight nodes having three degrees of freedom at each node and the orthotropic material properties. The default element coordinate system is along global directions. The element is usually used for modelling plastic elastic deformation, shrinkage, creep and initial stress, initial deformation, and large deformation. The truss element was selected for modelling the yarns, which are subjected to tensile loads only and do not transfer any type of moment $[28,29]$. As the truss element the Link $180-3 \mathrm{D}$ finite strain spar was used. This element is similar to link8 but with improved nonlinear capabilities. The curved truss element in 3D space has six deformation parameters in each node. The element's deformation parameter matrix has a total of twelve elements. This element transmits only the axial force, but it is deformed by bending and torsion. In technical practice, an uniform distribution of shear deformation on the cross-section originates is assumed for the truss element, but according to the Midlin hypothesis with possible deviation of the perpendicularity to the centerline of the element. Therefore, unlike for the straight bar (Link8), it was necessary to consider the curved truss element (Link180), which is based on the decomposition principle of the centerline elongation and the change of curvature.
For the static analysis, owing to the duration of the entire process, an automatic step control scheme was used. Generally, because of the small thickness of the fabric component, the stress in the thickness direction of the material can be neglected. Therefore, technical textiles are thought to be affected by plane stress.

\subsubsection{UD model}

Unidirectional loading was modelled using FEM in ANSYS. Owing to the complexity of the geometry and the lengthy calculation, the model was divided and solved in half axial symmetry. Gradually, the material properties of the fabric, structural steel, and PA fibre were introduced into the model (Table 3 ). For such a complicated geometry, it was necessary to create a mesh by using a combination of triangular polygons, geometric smoothing, and sweep methods.

In the critical parts of the geometry, the mesh of the thread was optimized using the Body Size function in the marked areas of the model. For body sizing, the definition of type element sizing $0.23 \mathrm{~mm}$ with coarse behaviour was introduced. In addition, the hex dominant method was used to generate the final mesh of the labelled geometry [30].

With regard to the same textile materials for all experiments, the same material model for multi axis simulation was used.

The FEM mesh was optimized so that individual elements did not overlap with the optimized meshes that were already created (Figure 7). In the Table 4 there is a specification of the optimized mesh.

Table 3. Material models used in unidirectional model

\begin{tabular}{|c|c|c|c|c|c|c|c|c|c|}
\hline \multirow{2}{*}{ Material } & & \multirow{2}{*}{ Density $\left[\mathrm{kg} \cdot \mathrm{m}^{-3}\right]$} & \multicolumn{2}{|c|}{$\begin{array}{l}\text { Elastic modulus } \\
{[\mathrm{GPa}]}\end{array}$} & \multicolumn{2}{|c|}{$\begin{array}{l}\text { Shear modulus } \\
{[\mathrm{GPa}]}\end{array}$} & \multicolumn{2}{|c|}{ Poisson ratio [-] } & \multirow{2}{*}{$\begin{array}{l}\text { Tensile } \\
\text { strength } \\
\text { [Gpa] }\end{array}$} \\
\hline & & & $F_{11}^{f, m}$ & $F_{22}^{f, m}$ & $G_{12}^{f, m}$ & $G_{23}^{f, m}$ & $V_{12}^{f, m}$ & $V_{23}^{f, m}$ & \\
\hline \multirow{3}{*}{ Uniaxial } & Fabric & 1250 & 4.9 & 4.4 & 1.96 & 1.1 & 0.32 & 0.35 & 2.1 \\
\hline & Structural steel & 7850 & 200 & & 76.9 & & 0.3 & & 0.25 \\
\hline & PA fibre & 1140 & 1.8 & & 0.74 & & 0.21 & & 0.23 \\
\hline
\end{tabular}




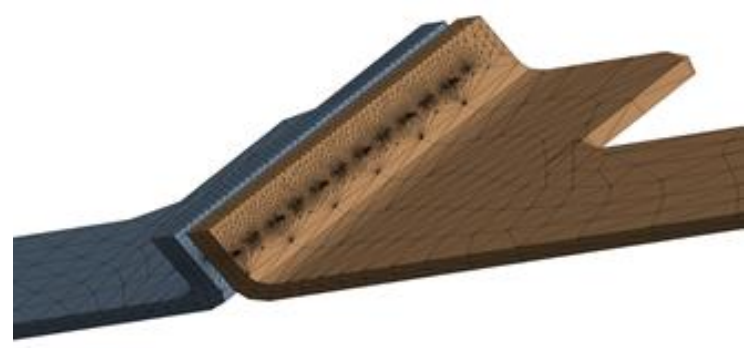

Figure 7. The mesh of the unidirectional model

Table 4. Specification of the created mesh in the UD model

\begin{tabular}{ccccc}
\hline The UD & $\mathbf{1}^{\text {st }}$ fabric & $\mathbf{2}^{\text {nd }}$ fabric & $\mathbf{1}^{\text {st }}$ thread & $2^{\text {nd }}$ thread \\
\hline Nr of nodes & 22797 & 27454 & 24864 & 16465 \\
Nr of elements & 101620 & 128000 & 17595 & 10637 \\
\hline
\end{tabular}

When two separate faces touch each other, they become tangential to each other and should therefore be considered in contact (Figure 8). They can transmit pressure normal forces and tangential friction. The higher the contact stiffness $\mathrm{K}$ [N m-1], the lower is the mutual penetration $\mathrm{x}$ [m]. The ideal case for infinite stiffness would have been zero penetration. However, when using Penalty Methods, this is numerically impossible [31]. In the real case, however, when the penetration $\mathrm{x}$ is very small, the result will still be sufficiently accurate. The frictional contacts with the coefficient $\mathrm{f}=0.3$ [32] was used for the modelled yarn (Figure 9a) and assembly completely (Figure 9b). The main difference, however, is the introduction of variable lambda, in which the Lagrangian method adds low sensitivity to the contact stiffness and this way provides an easier solution in the case of such complicated contacts. Another advantage of minor penetration is that when the penetration is not allowed, the contact is either opened or closed, that is, it can be represented using a step function. This can lead to problems in convergence or the solution oscillates between the open and closed statuses. Therefore, the basic Penalty Method was employed in the UD models. Normal stiffness is another essential contact parameter. In general, the higher the stiffness, the better is the accuracy.
However, solution convergence becomes difficult [29]. Concerning the definition of a contact pair, it is necessary to determine the distribution of stress in the two parts of the fibre. Therefore, for representing the mutual interactions of the fibres, symmetrical behaviour of the contact pair was selected. In a comparison of the interaction of yarn and fabric, it is possible to use automatic or asymmetric expressions. In the end, for derivation of integration points, the nodal-normal definition has been used [30]. The value of the maximum tensile stress depends on the displacement (Figure 17), as was observed in the results.

\subsubsection{Multiaxial model}

A smooth uniform mesh should be sought in the regions of interest for the analysis. Elsewhere, coarsening of the ambient mesh may help reduce the overall size of the problem to be solved [31]. The rigid and flexible parts of the model were meshed sequentially by using the sweep method in combination with triangular polygons. To optimize it, the mapping features were inserted. The input value of an element was set to $0.18 \mathrm{~mm}$. The mesh was optimized to the values specified in the Table 5.
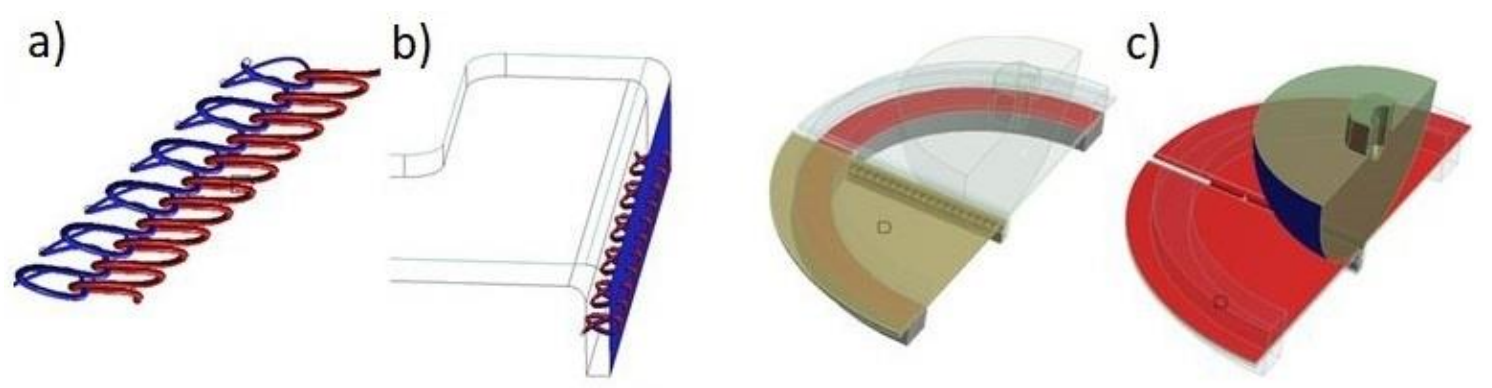

Figure 8. Scheme of solved numerical contact for uniaxial (a and b) and multiaxial (c) models 
a)

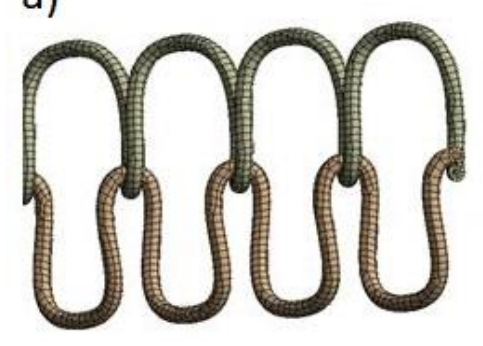

b)

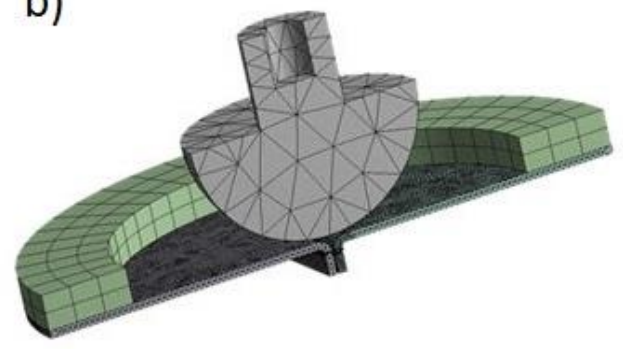

Figure 9. The mesh of the: a) sewing seam in detail b) whole multidirectional model

Table 5. Specification of the created mesh in the multiaxial model

\begin{tabular}{ccccccc}
\hline The multiaxial & Indentor & Jaw & 1st fabric & 2nd fabric & 1st thread & 2nd thread \\
\hline Nr of nodes & 12281 & 800 & 3650 & 3715 & 25402 & 13661 \\
Nr of elements & 2763 & 1275 & 1902 & 1915 & 8986 & 8913 \\
\hline
\end{tabular}

Between the rigid and flexible parts of the model, the friction was set. Selected contact surfaces were defined using the friction coefficient $\mathrm{f}=0.3$ and Normal Lagrange formulation (Figure 8c) [32]. After optimization of the contacts, the indenter, which was in contact with the tested fabric, was set to undergo remote displacement in one direction. The value of the maximum tensile stress depends on the displacement (Figure 22), as was observed in the results.

\subsubsection{The introduction of models boundary conditions}

For simulation of the both models (unidirectional and multiaxial), different boundary conditions were introduced. In the case of uniaxial loading modelling of at the bottom of the surface, the model was fixed in all movements and rotations. On the opposite side of the surface the displacement in uniaxial direction was set. This boundary condition has been defined to the maximum length in the displacement $\mathrm{u} \rightarrow$ depends on time. In the other directions, the displacements have been fixed to avoid some undesirable shift. The feed value $\mathrm{u} \rightarrow$ corresponds to the maximum elongation achieved during the real tests of the uniaxial fabric.

In case of the modelling of uniaxial loading at the bottom of the surface, the model was fixed for all movements and rotations. On the opposite side of the surface, the displacement along the uniaxial direction was set. This boundary condition was defined such that the maximum length along the displacement $\mathrm{u} \rightarrow$ depended on time. Along the other directions, the displacements were fixed to avoid any undesirable shift (Figure 10a). The feed value $u$ $\rightarrow$ corresponds to the maximum elongation achieved during real tests of the uniaxial fabric.

In the case of multiaxial loading modelling, the model was divided into two parts, namely, rigid and flexible. The rigid part of the model formed a jaw and indenter, into which the material properties of the structural steel were introduced. In the flexible parts of the model, the material properties of the fabric and PA 30 sewing threads were inserted (Figure $10 \mathrm{~b})$.
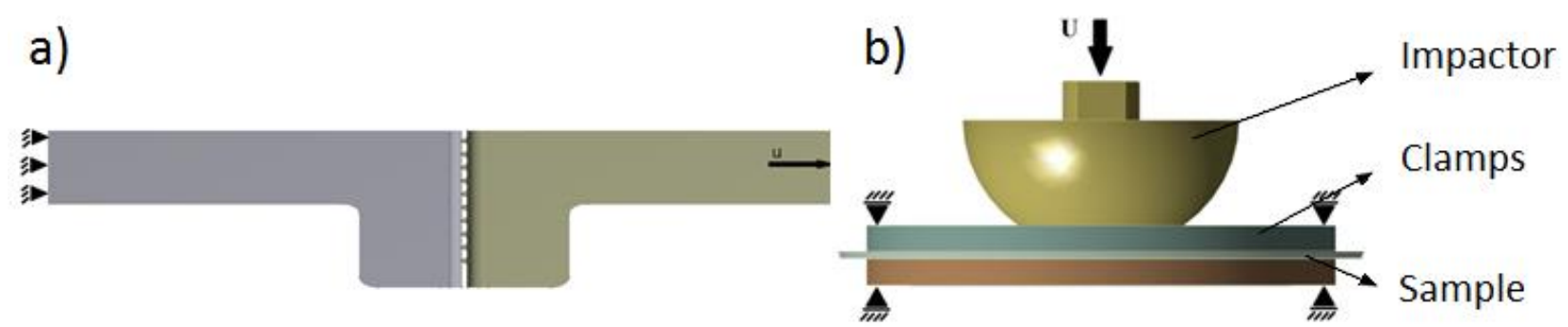

Figure 10. Defined boundary conditions: a) uniaxial measurements and b) multiaxial measurements 
To improve the efficiency of analyses involving large number of bodies, is advantageous to suppress the default frictionless interaction that is scoped to all bodies, and insert additional body interaction objects, which limit interactions to specific bodies instead. The value of the displacement $\vec{u}$ was the maximal value of displacement, equal to the on measured when the yarn was broken during the experiments. The determined value corresponds to mean values of the measured displacements during which breach of threads in real samples occurred. The observed results were the values of maximum tensile stress in dependency on the displacement (Figure 20). Two kinds of stepping control of the static analysis have been used. The first one with a constant time step size and the second with substeps adjustments. As could be seen in the Figure 11, the chosen approach has little bit changed the convergence of solution, however there was no impact to the duration or precision of the results.

The most important step to the successful convergence in the studied case were not any very precise mesh neither the setting of the solver, but definition of the contact, especially the detection of the integration points on the contact surfaces. This could be caused by the fact, that as could be seen in Figure 12 the contact is mostly sliding and the integration contact points varying for the each step. a)
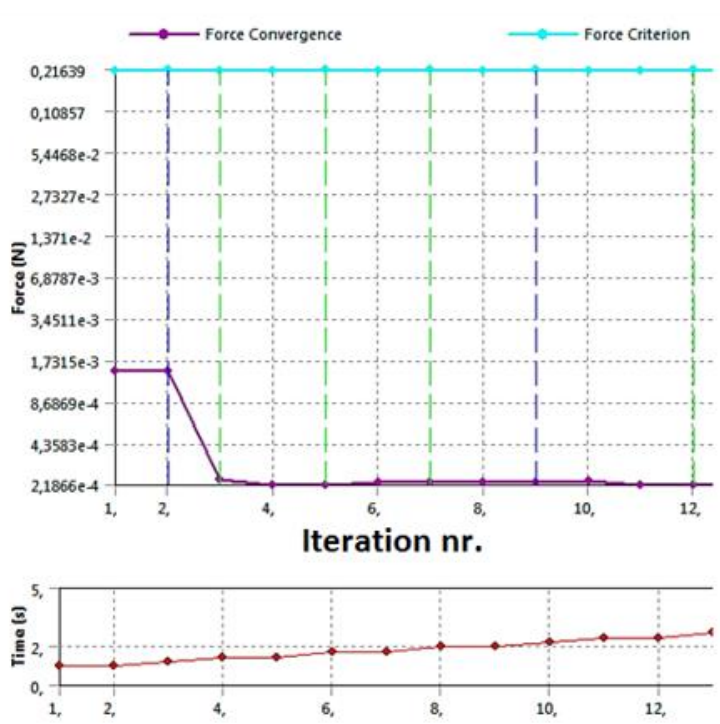

b)
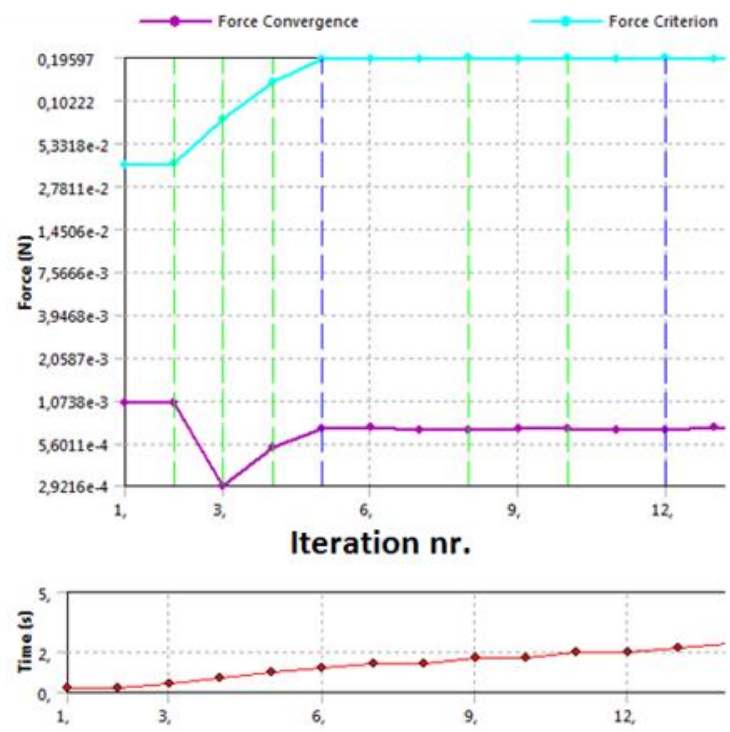

Figure 11. Convergence of the model solver for the a) Constant b) Variable step control

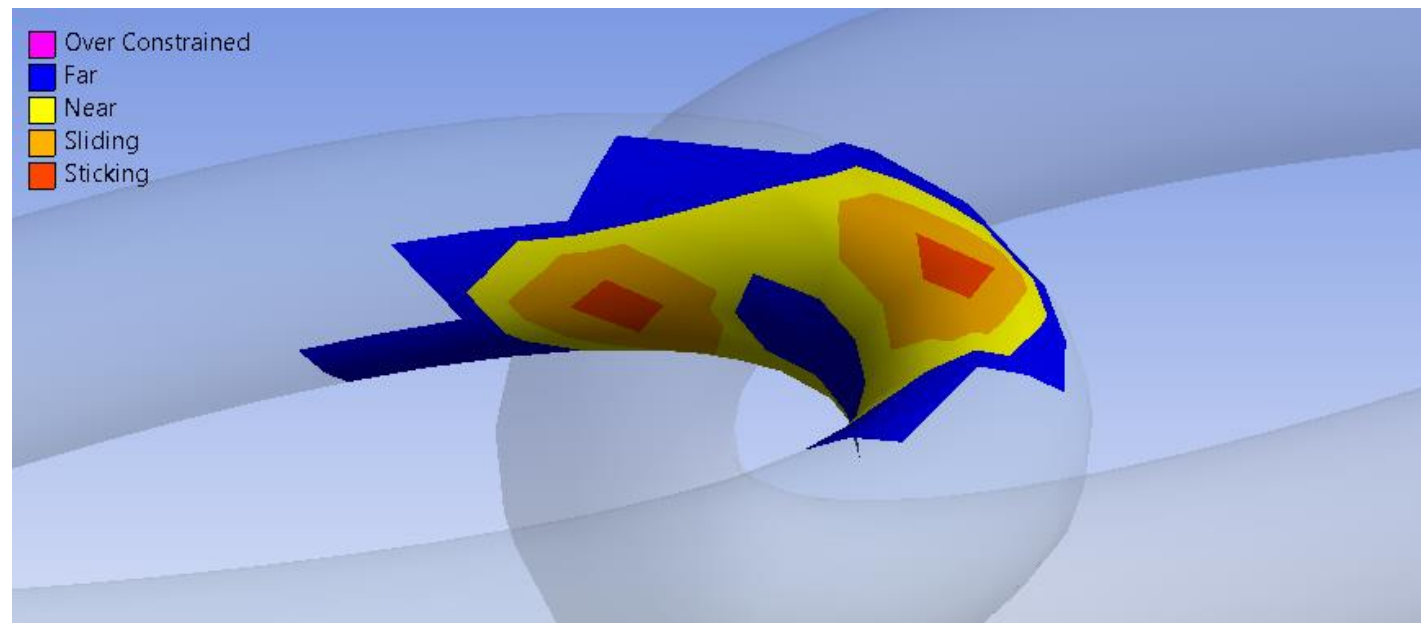

Figure 12. The status of the mutual threads contact 


\section{RESULTS AND DISCUSSION}

In this work, the mechanical characteristics of seams were analysed experimentally for various types of stresses. The device that can be used to test seam strength and monitor the mechanical characteristics of seams under multi-axial loading was developed. Then also the experimental analysis of the dynamic characteristics of the seam was performed to compare the strength of sewed seams under static and dynamic stresses.

\subsection{Experimental Results}

\subsubsection{Unidirectional tests}

The ultimate strength tests under different types of loading are summarized in Figure 13, where the loading curves are shown (as an example for seams with PA 30 thread). The fiber strength was determined based on the known cross section area, normal stress and the ultimate strength known from the experiments.

A correlation can be observed between the results of static loading in warp and weft directions. However, based on the experimental results it was found that for static loading in the warp direction, the maximal forces were approximately $10 \%$ higher than in the weft direction (Figure 14). In all seams with the various sewing threads were used. The material is strongest when the seam is perpendicular to the warp direction and weakest when the seam is perpendicular to the weft direction.
This is probably related to the fact that the density expressed by the number of threads per unit length is higher in warp direction ( 28 ends per $\mathrm{cm}$ and 22 picks per $\mathrm{cm}$ ).

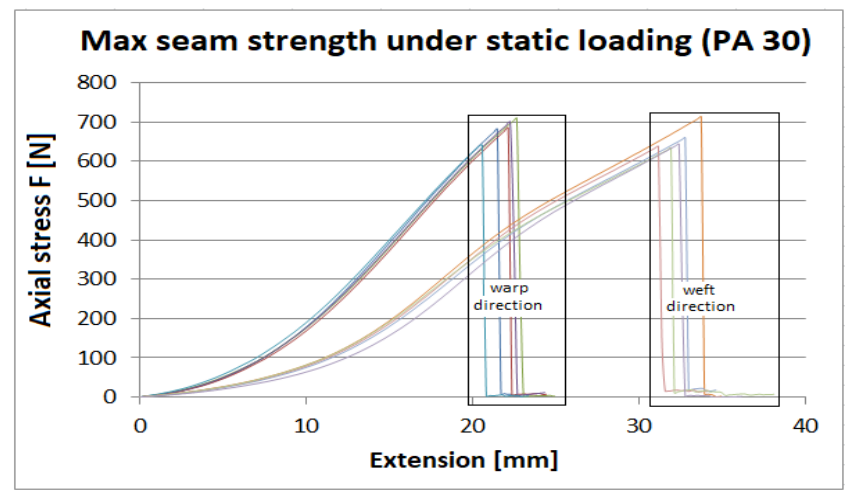

Figure 13. Force-elongation curves of seams with PA 30 threads in warp and weft directions (unidirectional static test)

\subsubsection{Multiaxial tests}

Four types of polyester and polyamide sewing threads were used for sewing the sandwich material, which is used directly for sewing car seat covers. The axial stress and elongation of seams under multiaxial stress were measured. The test results in the form of stress-strain curves for seams using PA 30 threads, as an example, are shown in Figures 15 and 16.
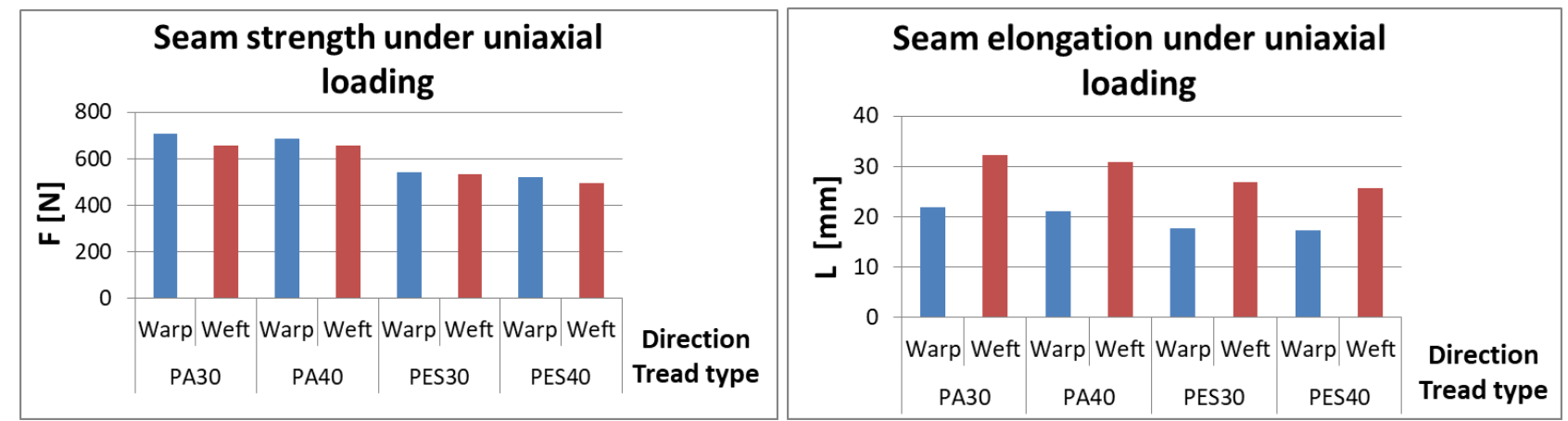

Figure 14. Changes in seam breaking forces and elongation depending on thread type and test direction

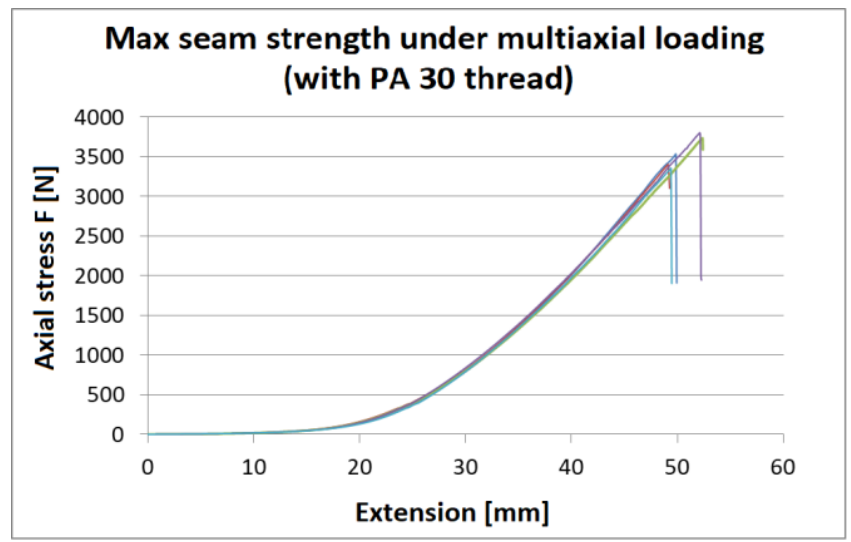

Figure 15. Axial stress to seam break under multiaxial load

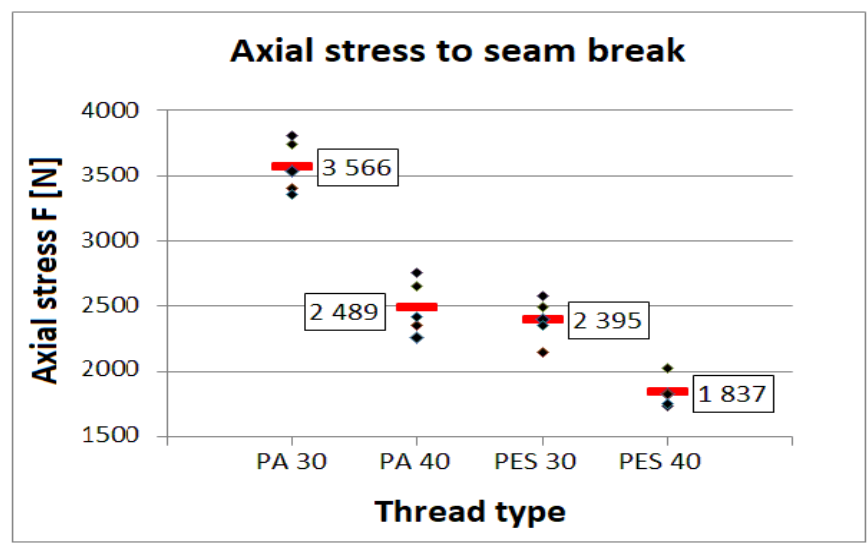

Figure 16. Experimentally determined value of axial stress to seam break with dependency on thread type under multiaxial stress (red lines are averages of axial stress to seam break) 


\subsection{Static Uniaxial Loading}

The first step before the main modelling was exploring how real seam rupture occurs. For better comprehension, seam rupture was monitored using high-speed cameras i-SPEED 3 over the course of the experiments. Examples of seam rupture observed sewed with PA30 thread type under uniaxial static loading are shown in Figure 17. In the image, locations of seam rupture are visible clearly at the recording speed of 10,000 fps for static loading.

The solution of this model was the maximum tensile stress observed in the added fabric yarns (Figure 18) and the resultant computed force compare with the experiment in the Figure 19.
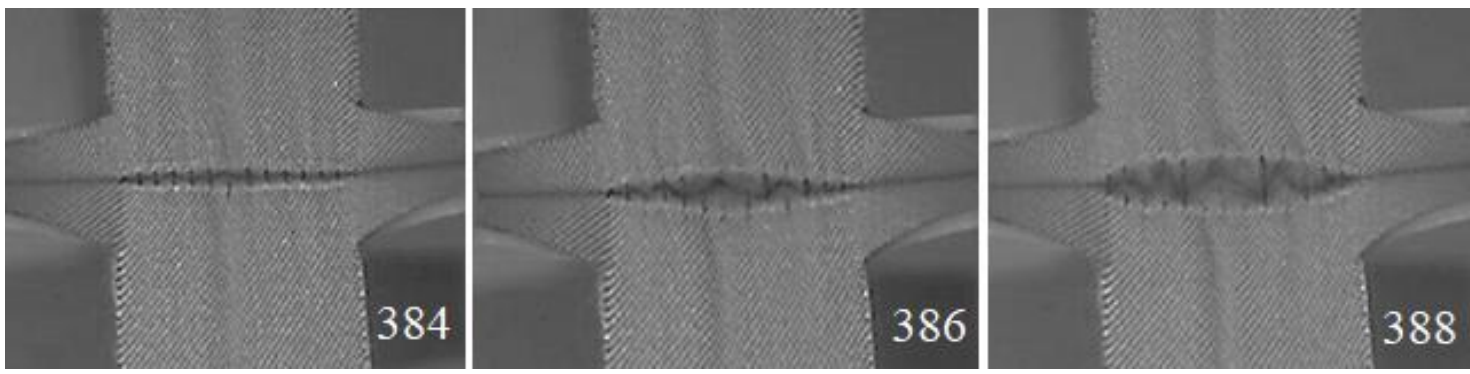

Figure 17. Moment of rupture under static uniaxial loading $\left(384^{\text {th }}\right.$ frame, $386^{\text {th }}$ frame, and $388^{\text {th }}$ frame $)$
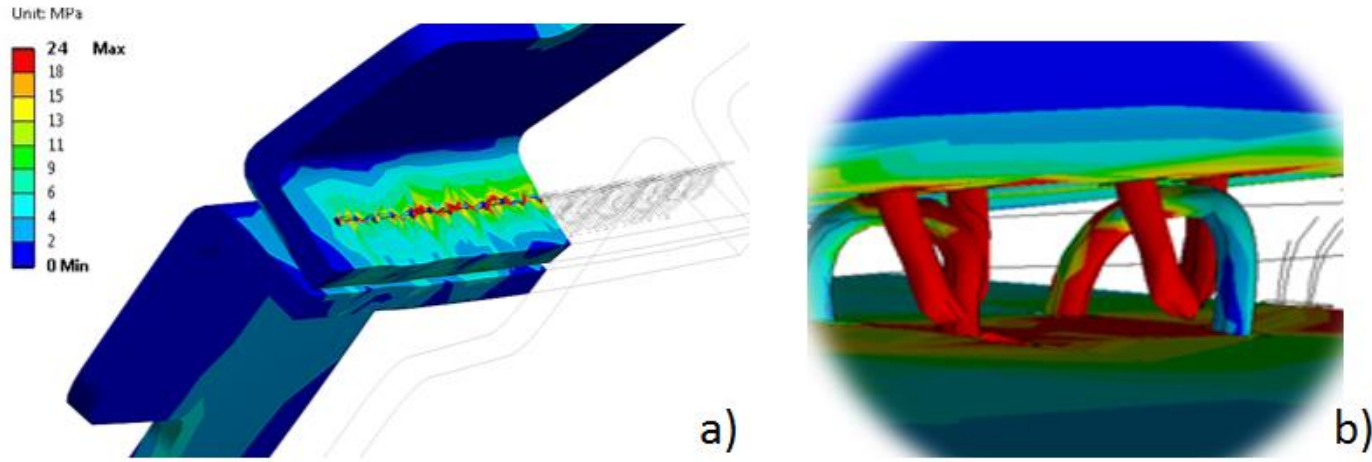

Figure 18. Normal stress in uniaxial model

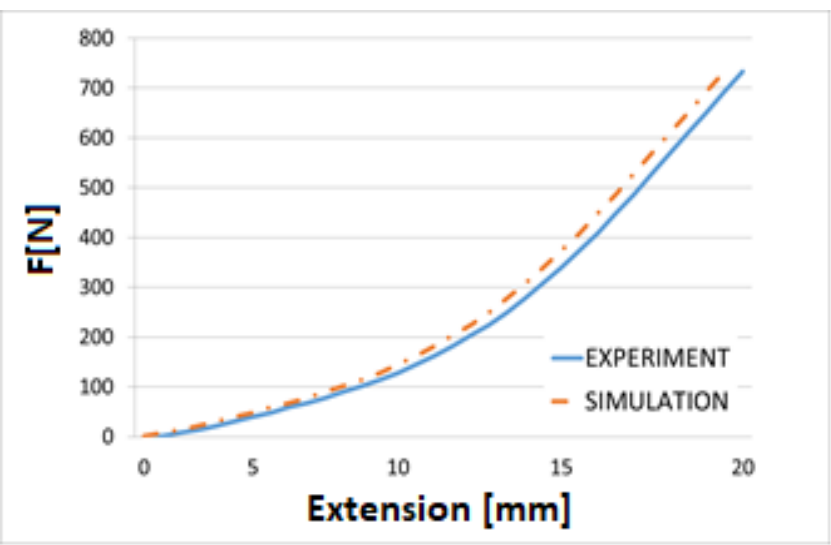

Figure 19. Results of for static loading

\subsection{Static Multiaxial Loading}

The location of rupture of the seam with PA30 thread type during multiaxial loading was monitored using a high-speed camera with a recording speed of 10,000 fps. Examples of seam rupture are shown in the Figure 20.

The maximum tensile stress observed in the added fabric yarns (Figure 21) together with the position of the indenter and the resultant computed forces are shown in Figure 22.

The results of the conducted experiment and the simulations under static unidirectional loading with PA 30 sewing thread in a seam were compared to each other (Figure 22). 

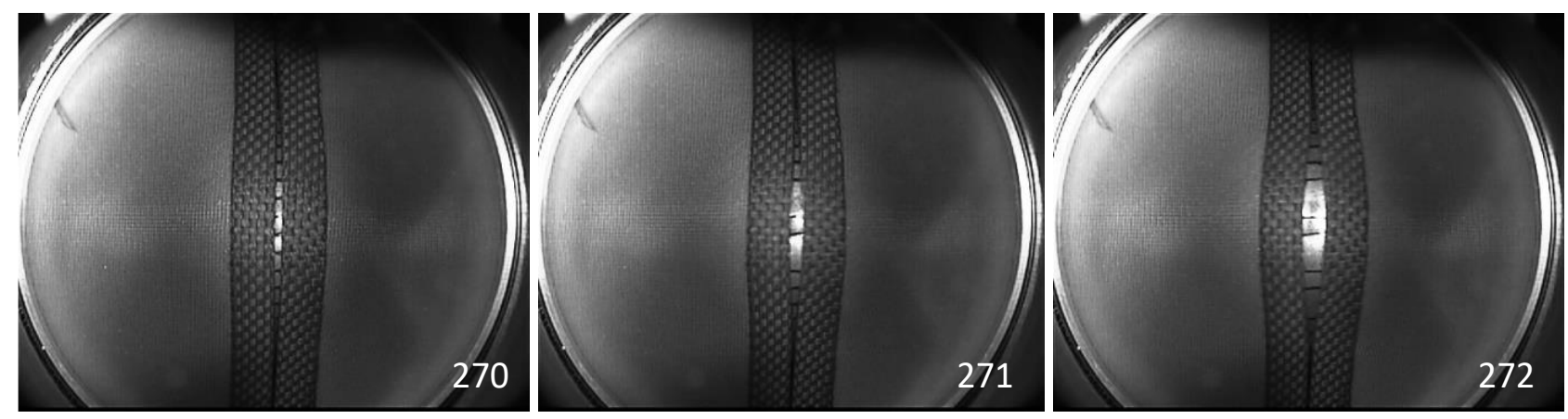

Figure 20. Moment of growing rupture under dynamic uniaxial loading

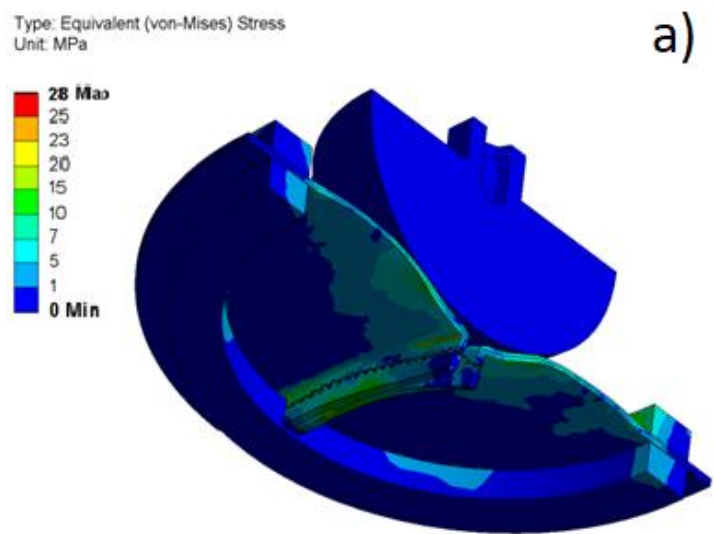

b)

Figure 21. Normal stress in uniaxial model

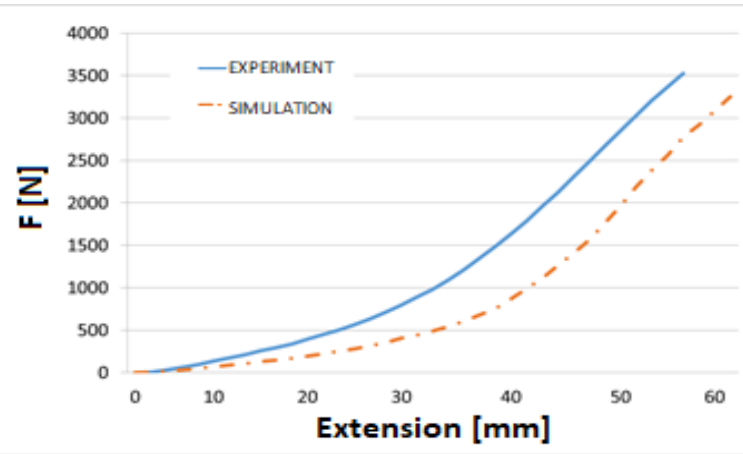

Figure 22. Results of model and experiment under multiaxial static loading

The outputs of the experiment for determining the strength of sewing seams in car seat covers revealed that seams with PA 30 threads have the highest strength and elongation, and they are the most suitable for sewing covers compared to other threads. In the simulation results, the maximum normal stress in the seam area could be observed. The simulated values correspond to the experimental values with a deviation of $8 \%$ in force and $10 \%$ in displacement.

\section{CONCLUSION}

In this study, a method of measuring the strength of sewing seams was proposed. The device for seam strength measurement under unidirectional and multiaxial stress in static and dynamic loading was developed. This is useful because of the anisotropy of sandwich structures in the technical textiles used, for example, in car seat covers.

Based on the experimental measurements, the mechanical properties of several combinations of yarns and fabrics were determined. To predict behaviour of the seam in real conditions and to verify the experimental results, the unidirectional and multiaxial tensile tests were simulated numerically. At first, the CAD model based on the real microstructure obtained by $\mathrm{CT}$ and micro capture, with the lockstitch threads connections, was designed and imported to ANSYS 18.0, where the model was subjected to real loading and boundary conditions. Based on the experiments, the material models of the yarns and fabric used herein were determined. The simulation results agree well with the results of the real tensile test. Based on these results, it can be said that the given sewing seams exceeded the limit of proportionality of $680 \mathrm{~N}$ in the in static mode, respectively, in the model of unidirectional loading, and this led to their rupture, as shown in Figure 18. Furthermore, the maximum tensile stress in the threads and the textile materials was investigated. The model of multiaxial stress of the seam yielded the maximum normal stress of $28 \mathrm{MPa}$, which corresponds approximately to 3500 $\mathrm{N}$, as shown in Figure 22.

Every model and technique has default estimations. In the unidirectional test the simulation overestimates the strength 
and on the multidirectional it underestimates it as seen on Figures 19 and 22. Additionally it differentiates more in the multidirectional test, what is primarily associated with a large number of parameters and contacts in multidirectional modeling. The predictability of mode is in the reasonably good, but it is necessary to find ways to improve the models. The solution of the problem of optimization of a mathematical models of sewing seam under different loadings is consist the following stages: the choice of an optimization method, a directed search for a combination of the values of the parameters of a mathematical model ensuring the achievement of the desired goals, the accuracy of the models in particular.

This work provides new insights into the physical and mechanical properties of sewing seams, which are especially useful for the production and testing of technical textiles. Actuality of work is due to the high demands on the quality of technical textile products especially the demands on the strength of the seams, as one of the most important properties. The high cost of testing already finished products necessitates determining the sewing seam strength theoretically or testing appropriate samples by using a convenient device. In the future, research is needed to improve the developed apparatus for measuring the strength of a seam under multiaxial strain, correcting the seam numerical model to shorten the development cycle, which consists of preparing sample prototypes, testing the prototypes, and re-sampling. Another task will be modification of the materials used, which consist of composite parts. The newly created material models will be subjected to testing to improve the parameters and strength of the existing seams.

\section{ACKNOWLEDGEMENT}

This publication was written at the Technical University of Liberec as part of the results of this project Of the Ministry of Education, Youth and Sports of the Czech Republic and the European Union - European Structural and Investment Funds in the frames of Operational Programme Research, Development and Education - project Hybrid Materials for Hierarchical Structures (HyHi, Reg. No. CZ.02.1.01-0.00.0-16_019/0000843) and through the financial support of the Ministry of Education, Youth and Sports of the Czech Republic and the European Union (European Structural and Investment Funds - Operational Programme Research, Development and Education) in the frames of the project "Modular platform for autonomous chassis of specialized electric vehicles for freight and equipment transportation", Reg. No. CZ.02.1.01/0.0/0.0/16_025/0007293.

\section{REFERENCES}

1. Shishoo R. 2008. Textile advances in the automotive industry. The Textile Institute. Boca Raton Press: Woodhead, ISBN 9781845693312.

2. Fung W. 2000. Textiles in automotive engineering. Cambridge: Woodhead, ISBN 1855734931.

3. Barbulov-Popov D, Cirkovic N, Stepanovic J. 2012. The Influence of Stitch Density and of the Type of Sewing Thread on Seam Strength TEM Journal 2, 104-110.

4. Mazari A, Kausik B, Havelka A. 2016. Prediction of needle heating in an industrial sewing machine Textile Research Journal 86(3), 302-310.

5. Zak J. 2015. Modelling of textile structures Fibres and Textiles Slovak University of Technology in Bratislava, Vol. 22, 2015, ISSN 1335-0617.

6. Midha VK, Chatopadhyay R, Kothari VK. 2009. Studies on the changes in tensile properties of sewing thread at different sewing stages Textile Research Journal. 79(13), 1155-1167.

7. Rudolf A, Gersak J. 2011. The effect of drawing on PET filament sewing thread performance properties Textile Research Journal $82(2), 148-160$

8. Gülşah P, Çeken F. 2009. Research on the breaking and tearing strength and elongation and elongation of automobile seat cover fabrics Textile Research Journal 79(1), 47-58.

9. Ujevic D, Kovacevic S. 2004. Impact of the seam on the properties of technical and nonwoven textiles for making car seat coverings International Nonwovens Journal 13(1), 31-41.

10. Germanova-Krasteva D, Petrov H. 2008. Investigation on the seam's quality by sewing of light fabrics International Journal of Clothing Science and Technology (20), 57-64.

11. Bagnaci B, Shanbeh M, Ghareaghaji A. 2010. Effect of tensile fatigue cyclic loads on bagging deformation of elastic woven fabrics Indian Journal of Fibre \& Textile Research 35, 298-302.
12. Quaglini V, Corazza C, Poggi C. 2008. Experimental characterization of orthotropic technical textiles under uniaxial and biaxial loading. Composites Part A Applied Science and Manufacturing 39(8), 1331-1342.

13. Kawabata S, Niwa M. 1984. Validity of the linearizing method for describing the biaxial stress-strain relationship of textile. The University of Shiga Prefecture.

14. Wang Y, Zhang P, Zhang Y. 2014. Experimental investigation the dynamic pressure attenuation of elastic fabric for compression garment Textile Research Journal [online] 84(6), 572-582 [cit. 201603-28].

15. ISO 2060: 1994. Textiles: Yarn from packages-Determination of linear density (mass per unit length) by the skein method.

16. ISO 2061: 2010. Textiles: Determination of twist in yarns - Direct counting method.

17. ISO 5084: 1996. Textiles-Determination of thickness of textile and textile products.

18. ISO 13935-1: 2014. Textiles - Seam tensile properties of fabrics and made-up textile articles - Part 1: Determination of maximum force to seam rupture using the strip method.

19. Kovalova N, Kulhavý P, Vosáhlo J, Havelka A. 2016. Numerical Model and Analysis of Multiaxial Stress on the Sewing seam. Proceeding of International Conference AUTEX. University of Ljubljana, Ljubljana.

20. Reinhard HW, Corazza C, Poggl C. 1976. On the biaxial testing and strength of coated fabrics Experimental Mechanics 16(2), 71-74.

21. Novak O, Hanuš J. 2010. 3D nonwovens in medicine-simulation of mattresses behavior under loading. Liberec: Technical university in Liberec, 112.

22. Escarpita D, Koenders EAB, Carvalho DBF. 2012. Biaxial tensile strength characterization of textile composite materials Composites and Their Properties. InTech, 08-22. 
23. Mukhopadhyay SK., Jeffrey FL. 1999. Partridge automotive textiles. The textile Institute, Oxford,

24. ISO 139: 2005. Textiles — Standard atmospheres for conditioning and testing.

25. Kulhavy P, Kovalova N, Vosahlo J. 2015. Numerical model of the static loading of a stitched seam in the composite cover of car seat Applied Mechanics and Materials 827, Trans tech publication.

26. Matousek I. 2005. Computer modelling in the automated production of molded glass. Technical University of Liberec: Liberec, 28s. ISBN 80-708-3988-0.

27. Zára J., Benes B. 2004. Modern computer graphics. Computer Press: Brno, 609 p. ISBN 80-251-0454-0.

28. Vosahlo J, Novak O, Petru M, Lepsik P. 2014. Experimental and numerical study of mechanical properties of artificial blood vessel, EAN- 52nd International Conference. on Exp. Stress Analysis. Marianske Lazne; Czech Republic, 175-176.
29. Madenci E, Guven I. 2006. The Finite Element Method and Applications in Engineering Using ANSYS. Springer, ISBN-13: 9780387282893

30. Talia M, Lankarani H, Talia JE. 1999. New experimental technique for the study and analysis of solid particle erosion mechanisms Wear pp. 225-229, pp.1070-1077.

31. Jirasek M, Bazant Z. 2002. Inelastic analysis of structures, Wiley, ISBN 0-471-98716-6.

32. Zavarise G, Wriggers P, Schrefler BA. 1995. On augmented Lagrangian algorithms for thermomechanical contact problems with friction International Journal for Numerical Methods in Engineering 38 (17), 2929-2949. 